\title{
Generation of twin Fock states via transition from a two-component Mott insulator to a superfluid
}

\author{
M. Rodríguez, S. R. Clark, and D. Jaksch \\ Clarendon Laboratory, University of Oxford, Parks Road, Oxford OX1 3PU, United Kingdom
}

(Received 15 July 2006; published 2 January 2007)

\begin{abstract}
We propose the dynamical creation of twin Fock states, which exhibit Heisenberg-limited interferometric phase sensitivities, in an optical lattice. In our scheme a two-component Mott insulator with two bosonic atoms per lattice site is melted into a superfluid. This process transforms local correlations between hyperfine states of atom pairs into multiparticle correlations extending over the whole system. The melting time does not scale with the system size which makes our scheme experimentally feasible.
\end{abstract}

DOI: $10.1103 /$ PhysRevA.75.011601

PACS number(s): 03.75.Dg, 03.75.Lm, 03.75.Mn, 05.30.Jp

Degenerate atomic Bose and Fermi gases [1] provide an excellent starting point for engineering almost pure manyparticle quantum states which are an essential resource for novel quantum technologies. This is illustrated by the realization of a Mott insulator (MI) [2] in the lowest Bloch band of an optical lattice which can serve as a quantum memory [3]. However, new methods are necessary for attaining some of the most important $N$-particle states, in particular those that allow a sensitivity enhancement from the standard quantum limit proportional to $1 / \sqrt{N}$ to the Heisenberg limit proportional to $1 / N$ in quantum metrology [4]. Here we propose a method for engineering twin Fock states [5] starting from a two-component MI with two bosonic atoms per site in an optical lattice $[3,6]$. Our scheme manipulates the hyperfine states $a$ and $b$ of atom pairs pinned to single lattice sites and decoupled from one another in the MI regime. In this limit the dynamics is reduced to a set of identical two-particle problems and correlations between states $a$ and $b$ are accurately induced using Raman laser pulses or microwaves and collisional interactions controlled via Feshbach resonances [7]. These local two-particle correlations are then transformed into multiparticle correlations extending over the whole system by melting the MI into a superfluid (SF). This quantum melting can be experimentally implemented by adiabatically ramping down the depth of the lattice potential [2] as shown in Fig. 1(a). By an appropriate choice of correlations created in the MI phase a twin Fock state emerges in the resulting two-component superfluid. Using this state in a Mach-Zehnder interferometer (MZI), shown in Fig. 1(b), one can approach sensitivities scaling as $1 / N$.

Our method is motivated by theoretical and experimental evidence $[2,8]$ that long-range correlations build up quickly when melting a MI to a SF. Almost adiabatic melting can be achieved with ramping times $t_{r}$ on the order of a few tens of $N / M J_{0}$ where $J_{0}$ is the typical tunneling amplitude between lattice sites during the melting process and $M$ is the number of lattice sites [8]. Importantly, in our scheme $M \propto N$, thus preventing $t_{r}$ from scaling with $N$. The achievement of sensitivities at the Heisenberg limit relies acutely on the relative number of atoms entering each port of the interferometer. This stringent requirement rules out simple schemes involving either a $\pi / 2$ rotation into a two-component BoseEinstein condensate (BEC) or a rapid splitting of a singlecomponent BEC in a double-well potential, since both result in a binomial relative number distribution. While number squeezing to a twin Fock state could be achieved in the latter case by an adiabatic splitting, this time scale $t_{r} \propto N$ since $M=2$ is fixed $[8,9]$. In contrast, the smaller $t_{r}$ for our scheme is less demanding on the suppression of dissipation which is known to degrade the achievable sensitivities [10,11]. We note another promising route to generating a twin Fock state through the coherent dissociation of a molecular BEC as suggested in [12].

Our starting point is a MI with two atoms in each lattice site $i$ given by $\left|\Psi_{a b}\right\rangle=\Pi_{i}|a b\rangle_{i}[6,13]$. This state, with exactly the same number of $a$ and $b$ atoms, can be created by collisional interactions involving an auxiliary state $c$ as experimentally demonstrated in [6]. We analyze the melting of $\left|\Psi_{a b}\right\rangle$, and also of the superposition state $\left|\Psi_{a a+b b}\right\rangle$ $=\Pi_{i}\left(|a a\rangle_{i}+|b b\rangle_{i}\right) / \sqrt{2}$ obtainable from $\left|\Psi_{a b}\right\rangle$ by applying a $\pi / 2$ Raman pulse. For this we use the two-component BoseHubbard model describing the dynamics of atoms trapped in the lowest Bloch band of a sufficiently deep optical lattice. The corresponding Hamiltonian is $(\hbar=1)$ [2]

$$
\begin{aligned}
\hat{H}= & -\sum_{\langle i, j\rangle}\left(J_{a} \hat{a}_{i}^{\dagger} \hat{a}_{j}+J_{b} \hat{b}_{i}^{\dagger} \hat{b}_{j}\right)+U \sum_{i} \hat{n}_{i}^{a} \hat{n}_{i}^{b}+\frac{V_{a}}{2} \sum_{i} \hat{n}_{i}^{a}\left(\hat{n}_{i}^{a}-1\right) \\
& +\frac{V_{b}}{2} \sum_{i} \hat{n}_{i}^{b}\left(\hat{n}_{i}^{b}-1\right),
\end{aligned}
$$

where $\hat{a}_{i}\left(\hat{b}_{i}\right)$ is the bosonic destruction operator for an $a(b)$ atom localized in lattice site $i, \hat{n}_{i}^{a}=\hat{a}_{i}^{\dagger} \hat{a}_{i}$, and $\hat{n}_{i}^{b}=\hat{b}_{i}^{\dagger} \hat{b}_{i}$, while $\langle i, j\rangle$ denotes summation over nearest neighbors. The parameter $J_{a(b)}$ is the tunneling matrix element for atoms in state $a$ (b); $V_{a(b)}$ and $U$ are the on-site intra- and interspecies inter-

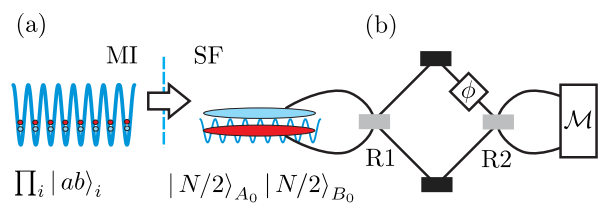

FIG. 1. (Color online) (a) Melting of a two-component twoatom MI into a SF. (b) The internal states $a$ and $b$ represent the arms of an interferometer with rotations R1, R2 and phase shift $\phi$ induced by a Ramsey pulse sequence; $\phi$ is measured by $\mathcal{M}$. 
action matrix elements, respectively. For simplicity we only consider the symmetric case with $J_{a(b)}=J, V_{a(b)}=V$. The ratio between the matrix elements is determined by the lattice depth [2] and additionally $U, V$ can be controlled via Feshbach resonances [7] or by shifting the $a$ and $b$ atoms away from each other using state-dependent lattices [3]. We denote the total number of atoms in state $a(b)$ as $\hat{N}_{a(b)}=\sum_{i} \hat{n}_{i}^{a(b)}$ and introduce Schwinger boson operators $\hat{J}_{x}=\sum_{i}\left(\hat{a}_{i}^{\dagger} \hat{b}_{i}+\hat{b}_{i}^{\dagger} \hat{a}_{i}\right) / 2$, $\hat{J}_{y}=\sum_{i}\left(\hat{a}_{i}^{\dagger} \hat{b}_{i}-\hat{a}_{i}^{\dagger} \hat{b}_{i}\right) / 2 i, \hat{J}_{z}=\left(\hat{N}^{a}-\hat{N}^{b}\right) / 2$, and $\hat{\mathbf{J}}=\left(\hat{J}_{x}, \hat{J}_{y}, \hat{J}_{z}\right)$.

We illustrate the outcome of melting two-component MI ground states with fixed total particle number $N=2 M$ assuming adiabatic evolution in an $M$-site system with periodic boundary conditions. The melting starts deep in the MI regime, where $J$ can be neglected. For $U<V$ the nondegenerate MI ground state is $\left|\Psi_{a b}\right\rangle$ and this adiabatically melts into the nondegenerate SF ground state $\left|\Psi_{\mathrm{tf}}\right\rangle=|N / 2\rangle_{A_{0}}|N / 2\rangle_{B_{0}}$ $\propto\left(\hat{A}_{0}^{\dagger}\right)^{N / 2}\left(\hat{B}_{0}^{\dagger}\right)^{N / 2} \mid$ vac $\rangle$ for $V / J \rightarrow 0$. Here all $N / 2 a$ atoms are in the same delocalized symmetric mode $\hat{A}_{0} \propto \sum_{i} \hat{a}_{i}$, all N/2b atoms are in mode $\hat{B}_{0} \propto \sum_{i} \hat{b}_{i}$, and $|\mathrm{vac}\rangle$ is the vacuum state. Thus, adiabatic melting of $\left|\Psi_{a b}\right\rangle$ provides a direct means of obtaining a twin Fock state $\left|\Psi_{\mathrm{tf}}\right\rangle$ with zero relative atom number difference.

In the opposite case $U>V$ the system exhibits spatial separation of the $a$ and $b$ components and has a large number of degenerate ground states. For an even number $N_{a}$ of $a$ atoms this degeneracy is lifted by completely connected hopping [14] and the state $\left|\Psi_{\text {sep }}^{N_{a}}\right\rangle$ $=S\left\{\Pi_{\left(i \leqslant N_{a} / 2\right)}|a a\rangle_{i} \Pi_{\left(N_{a} / 2<j \leqslant N\right)}|b b\rangle_{j}\right\}$ is adiabatically connected to the nondegenerate SF ground state $\left|N_{a}\right\rangle_{A_{0}}\left|N-N_{a}\right\rangle_{B_{0}}$, where $\mathrm{S}$ denotes symmetrization over lattice site configurations. Our second initial state $\left|\Psi_{a a+b b}\right\rangle$ is a binomial superposition of ground states $\left|\Psi_{\text {sep }}^{N_{a}}\right\rangle$ and therefore results in the melted state

$$
\left|\Psi_{\text {mac }}\right\rangle=\frac{1}{\sqrt{2^{N / 2}}} \sum_{m=0}^{N / 2}\left(\begin{array}{c}
N / 2 \\
m
\end{array}\right)^{1 / 2}|2 m\rangle_{A_{0}}|N-2 m\rangle_{B_{0}} .
$$

After rotation, the overlap of this state with the macroscopic superposition state [15] $\left|\Psi_{\max }\right\rangle=\left(|N\rangle_{A_{0}}|0\rangle_{B_{0}}+|0\rangle_{A_{0}}|N\rangle_{B_{0}}\right) / \sqrt{2}$ is found to be $\mathcal{O}=\left|\left\langle\Psi_{\max }\left|\exp \left(i \pi \hat{J}_{y} / 2\right)\right| \Psi_{\text {mac }}\right\rangle\right|=\sqrt[4]{8 / 9}>0.97$ for $N \rightarrow \infty$ and this limit is monotonically attained with $\mathcal{O}>0.97$ for $N>20$.

We can use either of the final states obtained by adiabatic melting to realize sensitivities proportional to $1 / N$ in the MZI setup shown in Fig. 1(b). For the twin Fock state $\left|\Psi_{\mathrm{tf}}\right\rangle$ R1 implements the conventional beam-splitter operation $\exp \left(i \pi \hat{J}_{x} / 2\right)$. In the case of $\left|\Psi_{\text {mac }}\right\rangle \mathrm{R} 1$ rotates the state according to $\exp \left(i \pi \hat{J}_{y} / 2\right)$ as discussed above. Then a relative phase $\phi$ is induced in one of the arms of the MZI. The operation R1 can be implemented by rapid resonant $\pi / 2$ Raman pulses, while the relative phase $\phi$ could be induced by an appropriate off-resonant pulse. The achievable phase sensitivity inside the interferometer (after R1) can be computed from the relative phase probability distribution $P(\theta)$. For a given state $|\psi\rangle=\sum_{m=0}^{N} C_{m}|m\rangle_{A_{0}}|N-m\rangle_{B_{0}}$ this is computed as $P_{s}(\Delta \theta)=\left|\sum_{m=0}^{N} C_{m} \exp (-i m \Delta \theta)\right|^{2} /(s+1)$ where $\Delta \theta$ is a

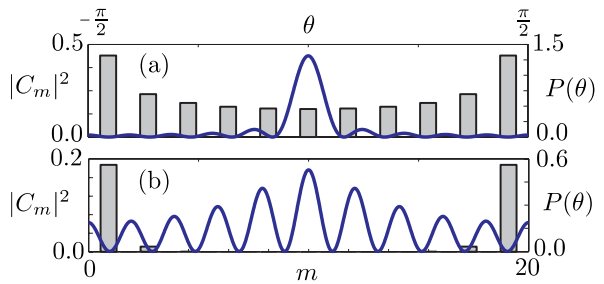

FIG. 2. (Color online) The amplitudes $\left|C_{m}\right|^{2}$ (left and bottom axes) and corresponding relative phase probability distribution $P(\theta)$ (right and top axes) after the rotation R1 of (a) $\left|\Psi_{\text {tf }}\right\rangle$ and (b) $\left|\Psi_{\text {mac }}\right\rangle$ with $N=20$.

multiple of $2 \pi /(s+1)$. The distribution $P(\theta)$ is obtained by multiplying $P_{s}(\Delta \theta)$ with $(s+1) / 2 \pi$ and taking the limit $s \rightarrow \infty$ [11]. As shown in Fig. 2(a) $P(\theta)$ is sharply peaked for the rotated $\left|\Psi_{\mathrm{tf}}\right\rangle$ and Fig. 2(b) displays the oscillatory behavior of $P(\theta)$ for the rotated $\left|\Psi_{\text {mac }}\right\rangle$. The sensitivity $\delta \phi$ of each state is quantified by the half width at half maximum of $P(\theta)$ around some fixed phase typically taken as $\theta=0$. From this definition it can be shown for both states that $\delta \phi \propto 1 / N$ and so scales at the Heisenberg limit $[5,11,15]$. The final step in the MZI consists of rotation $R_{2}$ and measurement $\mathcal{M}$ of a phase-dependent quantity which exhibits this Heisenberglimited phase resolution. For instance the measurement of parity as discussed in [16] or $\hat{J}_{z}^{2}$ as proposed in [17] can achieve this. These methods exploit nonlinearities caused by atomic collisions and are thus realizable in our setup.

We now analyze the achievable sensitivity for incomplete melting at finite (residual) values of $V / J$ before considering experimental imperfections arising from nonadiabatic ramping and particle loss. We restrict our considerations to the measurement of $\hat{J}_{z}^{2}$ and to the twin Fock state due to its greater experimental feasibility. We study these effects in terms of the noise $\Delta \phi$ on the $\phi$-dependent observable $\hat{J}_{z}^{2}$. Error propagation theory gives $\Delta \phi=\Delta \hat{J}_{z}^{2} /\left|\partial\left\langle\hat{J}_{z}^{2}\right\rangle / \partial \phi\right|$ where $\left\langle\hat{J}_{z}^{2}\right\rangle$ and $\Delta \hat{J}_{z}^{2}$ are the average and the spread, respectively. For states that are zero eigenvectors of $\hat{J}_{z}$, such as $\left|\Psi_{\mathrm{tf}}\right\rangle$, this gives

$$
\Delta \phi^{2}=\frac{\sin ^{2} \phi\left(\left\langle\hat{J}_{x}^{4}\right\rangle-\left\langle\hat{J}_{x}^{2}\right\rangle^{2}\right)+\cos ^{2} \phi\left\langle\hat{J}_{x} \hat{J}_{z}^{2} \hat{J}_{x}\right\rangle}{4 \cos ^{2} \phi\left\langle\hat{J}_{x}^{2}\right\rangle^{2}} .
$$

At $\phi=0$ the sensitivity reduces to $\Delta \phi(0)=\frac{1}{2}\left\langle\hat{J}_{x}^{2}\right\rangle^{-1 / 2}$ which can be expressed entirely in terms of the one-particle density matrices $\rho_{i j}^{a}=\left\langle\hat{a}_{i}^{\dagger} \hat{a}_{j}\right\rangle$ and $\rho_{i j}^{b}=\left\langle\hat{b}_{i}^{\dagger} \hat{b}_{j}\right\rangle$ using $\left\langle\hat{J}_{x}^{2}\right\rangle$ $=\left[\sum_{i}\left(\rho_{i i}^{a}+\rho_{i i}^{b}\right)+\sum_{i, j}\left(\rho_{i j}^{a} \rho_{j i}^{b}+\right.\right.$ H.c. $\left.)\right] / 4$. The initial MI state $\left|\Psi_{a b}\right\rangle$ with no off-diagonal correlations $\rho_{i j}^{a}=\rho_{i j}^{b}=\delta_{i j}$ yields the standard quantum limit $\Delta \phi=1 / \sqrt{2 N}$ [see Fig. 3(a) at $V / J \gg 1$ ]. The final SF $\left|\Psi_{\mathrm{sf}}\right\rangle$ with long-range correlations $\rho_{i j}^{a}=\rho_{i j}^{b}=1$ asymptotically recovers the Heisenberg limit $\Delta \phi$ $=1 / \sqrt{N^{2} / 2+N}$ [see Fig. 3(a) at $V / J=0$ ]. Thus the scaling $\Delta \phi \propto N^{-\alpha}$ changes from $\alpha=1 / 2$ to $\alpha=1$ during the melting.

The presence of a residual intraspecies interaction $V$ results in quantum depletion of the populations in the $A_{0}$ and $B_{0}$ modes, which reduces the attainable sensitivity. We con- 


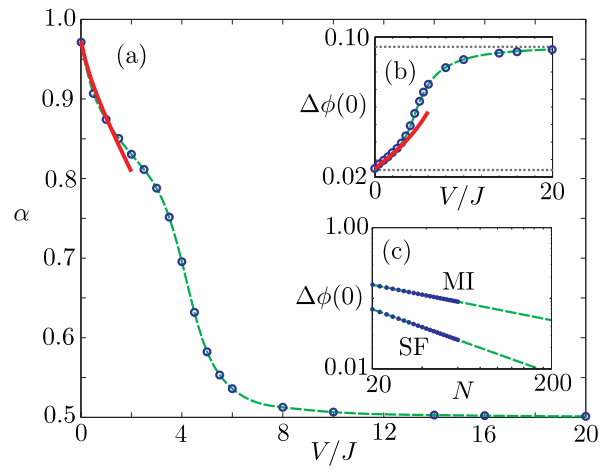

FIG. 3. (Color online) (a) Scaling $\alpha$ in $\Delta \phi \sim N^{-\alpha}$ against $V / J$ of ground states of $\hat{H}$ with $U=0$. The scaling was obtained from calculations with $N=20$ up to 60 with $M=N / 2$ for both the $1 \mathrm{D}$ numerical results $(\bigcirc)$ in a box system and the $N$-conserving Bogoliubov results (solid line). (b) Sensitivity $\Delta \phi$ as a function of $V / J$ for $N=60$. The dotted lines show exact results for the twin Fock SF (bottom) and the MI (top) states, while the thick solid line shows the Bogoliubov result. (c) $\Delta \phi$ as a function of $N$ for $V / J=20$ (MI) and $V / J=1 / 2$ (SF) giving $\alpha$ of the corresponding points in (a). In all cases the dashed lines are to guide the eye.

sider this effect for $U=0$, achieved for example by fully separating the sites of a spin-dependent lattice. In the SF regime $J \gg V$ a translationally invariant system is well described by the $N$-conserving Bogoliubov wave function

$$
\left|\psi_{\text {Bog }}\right\rangle \sim\left(\hat{\Lambda}_{a}\right)^{N / 2}\left(\hat{\Lambda}_{b}\right)^{N / 2}|\mathrm{vac}\rangle,
$$

where $\hat{\Lambda}_{a}=\left(\hat{A}_{0}^{\dagger 2}-\Sigma_{\mathbf{q} \neq \mathbf{0}} c_{\mathbf{q}} \hat{A}_{\mathbf{q}}^{\dagger} \hat{A}_{-\mathbf{q}}^{\dagger}\right)$ with $\hat{A}_{\mathbf{q}}$ the $\mathbf{q}$ quasimomentum modes for $a$, and similarly $\hat{\Lambda}_{b}$ is defined in terms of $\hat{B}_{\mathbf{q}}$ for component $b$. Within this ansatz the depletion is identical for both components and is given by $\zeta=\Sigma_{\mathbf{q} \neq \mathbf{0}} n_{\mathbf{q}}$ $=c_{\mathbf{q}}^{2} /\left(1-c_{\mathbf{q}}^{2}\right)$ where $n_{\mathbf{q}}=\left\langle\hat{A}_{\mathbf{q}}^{\dagger} \hat{A}_{\mathbf{q}}\right\rangle=\left\langle\hat{B}_{\mathbf{q}}^{\dagger} \hat{B}_{\mathbf{q}}\right\rangle$ and the amplitudes $c_{\mathbf{q}} \ll 1$ can be solved in terms of $V / J$ and are given in [18]. The sensitivity is then $\Delta \phi(0)=1 /\left[N^{2}(1-\zeta)^{2} / 2+N\right.$ $\left.+2 \Sigma_{\mathbf{q} \neq \mathbf{0}} n_{\mathbf{q}}^{2}\right]^{1 / 2}$. For a three-dimensional (3D) optical lattice the depletion $\zeta=(V / J)^{3 / 2} / 3 \pi^{2} \sqrt{2}$ and the sum $\Sigma_{\mathbf{q} \neq \mathbf{0}} n_{\mathbf{q}}^{2}$ $=(V / J)^{3 / 2}(3 \pi-8) / 12 \sqrt{2}$ are constant in $N$. Within the region of validity of the Bogoliubov ansatz there will thus be no degradation of the sensitivity scaling $\alpha$. Similarly we find no decrease of $\alpha$ in 2D within the Bogoliubov ansatz. In contrast, for a 1D system the depletion increases with $N$ since there is no true condensate for nonzero $V$ and long-range correlations decay algebraically. Consequently the scaling $\alpha$ decreases with increasing $V / J$ as shown in Fig. 3(a) for a finite number of particles.

We explore the sensitivity scaling between the SF and MI limit in 1D numerically using the time-evolving block decimation algorithm [19]. For specific values of $N$ we compute the ground states of the Hamiltonian Eq. (1) over a range of $V / J$ and $U=0$ and calculate their sensitivities. The result for $N=60$ in Fig. 3(b) shows a smooth transition between the ideal SF and MI results and agrees with the Bogoliubov result for $V / J \leqslant 2$. By repeating this calculation for different $N$ we extract the sensitivity scaling $\alpha$. For all values of $V / J$ the numerical results are well approximated by a power law

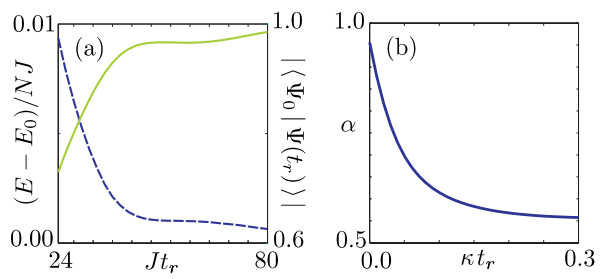

FIG. 4. (Color online) (a) Energy difference per atom $\left(E-E_{0}\right) / N J$ between $\left|\Psi\left(t_{r}\right)\right\rangle$ and the final SF ground state $\left|\Psi_{0}\right\rangle$ (left axis, dashed lines) and overlap $\left|\left\langle\Psi\left(t_{r}\right) \mid \Psi_{0}\right\rangle\right|$ (right axis, solid line) as a function of the ramping time $t_{r}$. Starting from the MI ground state at $V_{0} / J=20$ we ramped $V(t)$ down to a final $V_{t_{r}} / J=1 / 2$ using different ramping times $t_{r}$ and $U=0, N=50$, and $M=25$. (b) Scaling $\alpha$ of $\delta \phi$ with $N$ for $\left|\Psi_{\mathrm{tf}}\right\rangle$ under the presence of atom loss at a rate $\kappa$ obtained from results for $N=20$ up to 60 . Note that $\alpha$ does not reach unity because of the finite $N$ used.

$\Delta \phi \sim N^{-\alpha}$ as shown for the extreme cases in Fig. 3(c). We combine these results in Fig. 3(a) which displays the scaling $\alpha$ as a function of $V / J$. The value of $\alpha$ decreases smoothly from the Heisenberg limit in the SF regime to the standard quantum limit in the MI regime. As also shown in Fig. 3(a) the 1D Bogoliubov ansatz Eq. (3) is consistent with the numerics for $V / J \leqslant 2$ and the values of $N$ used here. In the MI regime a number-conserving particle-hole ansatz [20] underestimates long-range correlations and therefore does not predict an increase of $\alpha$ from $1 / 2$ at finite $V / J$. This emphasizes the importance of the growth of long-range correlations for increasing $\alpha$ above the standard quantum limit. While our numerical calculations are performed for sizes of $1 \mathrm{D}$ optical lattices currently used in experiments [2] we expect the scaling $\alpha$ to drop from $\alpha=1$ to $1 / 2$ at vanishingly small $V / J$ when $N \rightarrow \infty$ because of the lack of true long-range correlations. In $2 \mathrm{D}$ and $3 \mathrm{D}$ this reduction is instead expected to occur at finite $V / J$ close to the phase transition point $(V / J)_{\text {crit }}$ below which long-range correlations exist at large $N$.

In experiments the ramping time $t_{r}$ will be finite and so the melting is never perfectly adiabatic. We numerically compute the dynamical ramping of $V / J$ for the Hamiltonian Eq. (1) with $U=0$ from the MI ground state. In Fig. 4(a) the energy difference per atom between the final ramped state $\left|\Psi\left(t_{r}\right)\right\rangle$ and the SF ground state $\left|\Psi_{0}\right\rangle$ is shown as a function of the ramping time $t_{r}$, along with the corresponding manybody overlap $\left|\left\langle\Psi\left(t_{r}\right) \mid \Psi_{0}\right\rangle\right|$. For the infinite Bose-Hubbard model it has been shown [8] that $t_{r} \gg V_{\max } / J^{2}$ guarantees adiabatic evolution. Our numerics of a finite-sized system agree with this result, giving a near-unit overlap and small energy differences for ramping times of $t_{r} \sim 3 V_{0} / J^{2}$ with $V_{0}$ the initial interaction strength in the MI.

Given that the ramping time $t_{r}$ is much longer than the time required to implement the MZI in Fig. 1(b) we consider the influence of atom loss over a time $t_{r}$. The buildup of long-range correlations during the melting process has been found to be robust to atom loss processes [2]. However, atom loss will cause uncertainty in the relative number of $a$ and $b$ atoms in the final state $\left|\Psi_{\mathrm{tt}}\right\rangle$. We use the model introduced in [11] to calculate the sensitivity scaling $\alpha$ assuming that the state $\left|\Psi_{\text {tf }}\right\rangle$ is subject to atom loss at a rate $\kappa$ for time $t_{r}$. As shown in Fig. 4(b) we find that $\alpha \approx 1$ for $\kappa t_{r} \ll 0.1$. In com- 
bination with the adiabaticity condition on $t_{r}$ Heisenberglimited sensitivities can thus be achieved for $\kappa \ll J^{2} / 30 V_{0}$. This condition is within the reach of current optical lattice technology where tunneling amplitudes $J$ of a few hundred hertz and loss rates on the order of hertz can be achieved [2]. Furthermore, additional fluctuations in the overall particle number $N$ which occur from shot to shot do not affect the scaling $\alpha$ [17]. In the experiment a harmonic trapping potential is likely to be present and causes an outer shell of singly occupied lattice sites with atoms in the auxiliary state $c$. Additionally, an imperfect Raman transition during the creation of the initial MI state may leave a $c$-atom pair within the $\left|\Psi_{a b}\right\rangle$ state. In both cases the presence of a small number of $c$-atom impurities will not destroy the coherence of the final $\mathrm{SF}$ state, as seen in recent experiments [21]. Also these atoms will remain in state $c$ throughout the whole process and thus will not contribute to the particle number fluctuations [6].

In summary we have shown that quantum melting of a two-component $\mathrm{MI}$ in an optical lattice provides a viable route to engineer twin Fock states on time scales $t_{r}$ smaller than current experimental atom loss times. In particular the creation time $t_{r}$ does not scale with $N$. Our scheme also exploits the accurate controllability of two atom in a single lattice site to minimize fluctuations in the relative numbers of $a$ and $b$ atoms, which is crucial for achieving Heisenberglimited sensitivities proportional to $1 / N$.

We acknowledge fruitful discussions with I. Bloch and C. Simon. This work is supported by the EPSRC project EP/ C51933/1 and the EU project OLAQUI.
[1] L. Pitaevskii and S. Stringari, Bose-Einstein Condensation (Oxford University Press, Oxford, 2003).

[2] M. Greiner et al., Nature (London) 415, 39 (2002); T. Stöferle et al., Phys. Rev. Lett. 92, 130403 (2004); D. Jaksch et al., ibid. 81, 3108 (1998); S. R. Clark and D. Jaksch, Phys. Rev. A 70, 043612 (2004).

[3] O. Mandel et al., Nature (London) 425, 937 (2003).

[4] V. Giovanetti, S. Lloyd, and L. Maccone, Science 306, 1330 (2004), and references therein.

[5] M. J. Holland and K. Burnett, Phys. Rev. Lett. 71, 1355 (1993); P. Bouyer and M. A. Kasevich, Phys. Rev. A 56, R1083 (1997).

[6] A. Widera et al., Phys. Rev. Lett. 95, 190405 (2005); F. Gerbier et al., Phys. Rev. A 73, 041602(R) (2006); G. Thalhammer et al., Phys. Rev. Lett. 96, 050402 (2006).

[7] E. A. Donley, N. R. Claussen, S. T. Thompson, and C. E. Wieman, Nature (London) 417, 529 (2002); A. Marte et al., Phys. Rev. Lett. 89, 283202 (2002); M. Theis et al., ibid. 93, 123001 (2004).

[8] J. Dziarmaga et al., Phys. Rev. Lett. 88, 167001 (2002).

[9] G.-B. Jo et al., e-print cond-mat/0608585, and references therein.

[10] S. F. Huelga et al., Phys. Rev. Lett. 79, 3865 (1997).

[11] J. A. Dunningham, K. Burnett, and S. M. Barnett, Phys. Rev.
Lett. 89, 150401 (2002); S. M. Barnett and D. T. Pegg, Phys. Rev. A 42, 6713 (1990).

[12] K. V. Kheruntsyan and P. D. Drummond, Phys. Rev. A 66, 031602(R) (2002).

[13] P. Rabl et al., Phys. Rev. Lett. 91, 110403 (2003); M. Popp, J. J. Garcia-Ripoll, K. G. H. Vollbrecht, and J. I. Cirac, Phys. Rev. A 74, 013622 (2006).

[14] M. P. A. Fisher et al., Phys. Rev. B 40, 546 (1989).

[15] J. J. Bollinger, W. M. Itano, D. J. Wineland, and D. J. Heinzen, Phys. Rev. A 54, R4649 (1996).

[16] R. A. Campos, C. C. Gerry, and A. Benmoussa, Phys. Rev. A 68, 023810 (2003); C. C. Gerry and R. A. Campos, ibid. 68, 025602 (2003).

[17] J. A. Dunningham and K. Burnett, Phys. Rev. A 70, 033601 (2004); T. Kim et al., ibid. 60, 708 (1999).

[18] A. J. Leggett, Rev. Mod. Phys. 73, 307 (2001); M. Girardeau and R. Arnowitt, Phys. Rev. 113, 755 (1959); C. W. Gardiner, Phys. Rev. A 56, 1414 (1997).

[19] G. Vidal, Phys. Rev. Lett. 93, 040502 (2004); F. Verstraete, J. J. Garcia-Ripoll, and J. I. Cirac, ibid. 93, 207204 (2004).

[20] F. M. Cucchietti, B. Damski, J. Dziarmaga, and W. H. Zurek, e-print cond-mat/0601650.

[21] K. Günter et al., Phys. Rev. Lett. 96, 180402 (2006); S. Ospelkaus et al., ibid. 96, 180403 (2006). 